\title{
Las redes sociales de L'ETNO. Museu Valencià d'Etnologia. De la periferia al centro de la comunicación institucional
}

The social networks of L'ETNO. Valencià d'Etnologia Museum. From the periphery to the center of institutional communication

\author{
Francesc Cabañés Martínez ${ }^{\text {a }}$ y Remedios Garcia Curiel ${ }^{\text {b }}$ \\ ${ }^{a}$ Museu Valencià d'Etnologia, L'ETNO, fcabanes@dival.es ${ }^{\text {b }}$ Museu Valencià d'Etnologia, L'ETNO, \\ regacu@gmail.com
}

\begin{abstract}
Resumen
L'ETNO. Museu Valencià d'Etnologia es un museo público dedicado al estudio de la cultura tradicional y popular valenciana desde una perspectiva actual. Recientemente ha inaugurado la exposición permanente No és fàcil ser valencià/na, en la que se aborda la complejidad de la identidad valenciana desde tres ámbitos geográficos: la ciudad y la relación entre lo global y lo local; las huertas y marjales como espacios de imaginarios y tópicos valencianos; y el secano y la montaña, ámbito de las invisibilidades, fenómenos sociales existentes pero invisibles para la población urbana.
\end{abstract}

Como la mayoría de las instituciones de su ámbito, el museo tiene una escasa trayectoria en el universo de las redes sociales. L'ETNO puso en marcha sus redes en 2012 con la apertura de sus perfiles en YouTube y Flickr. Desde entonces ha abierto nuevos perfiles en Facebook, Twitter e Issuu (2013), Pinterest (2015) e Instagram (2017).

El presente texto tiene por objetivo analizar la trayectoria de estos canales y constatar su paulatina importancia dentro de la política de la comunicación institucional del museo. Estos canales percibidos como marginales en sus inicios han ganado centralidad y están propiciando cierta transformación en la conceptualización, planificación y edición de sus contenidos.

La función actual de las redes nos ha obligado a cambiar la forma de percibirlas: ya no son solo medios de información-como otros canales al uso (prensa y medios de comunicación)- o espacios de escucha, son herramientas de divulgación cultural y lugares de participación, lo que nos obliga a concebirlas como herramientas que afectan a todas las áreas del museo, no solo al departamento de comunicación.

Nuestra aportación pretende contar la experiencia concreta de cómo gestionar unos instrumentos que nos permiten informar de quiénes somos y qué hacemos, 
escuchar y ampliar nuestras audiencias y propiciar que éstas se conviertan en visitantes y participantes de nuestras actividades.

Por último, el texto se detendrá en algunos aspectos que están afectando a la gestión de estos canales, y que en nuestra opinión son básicos para afrontar el futuro: el incremento de estrategias y acciones que propicien la participación en línea de nuestros públicos, y la planificación y producción de contenidos digitales emanados desde la parte científica y educativa de la institución. Dos retos que suponen la diseminación del museo en territorios que superan la tradicional forma de consumir contenidos patrimoniales.

Palabras clave: museo, cultura tradicional, comunicación cultural, contenido digital, redes sociales, patrimonio cultural.

\footnotetext{
Abstract

L'ETNO, Valencian Museum of Ethnology is a public museum devoted to the study of popular and traditional valencian culture. The museum uses a contemporary approach which can be seen in the recently inaugurated permanent rooms titled "Not Easy to be Valencian". In this exhibition the museum proposes a critical perspective on valencian identity from three corners: the relation between the local and the global, the construction of cultural imaginaries and the existence of cultural invisibilities.
}

As most institutions of its kind, the museum has not a long experience with social media. L'ETNO started to develop its social media strategy in 2012 while opening accounts in YouTube and Flicker platforms. Since then it has expanded its presence to Facebook, Twitter and Issuu (2013), Pinterest (2015) and Instagram (2017)

This paper pretends to analyze the increasing weight of these social media channels into the institutional communication policies of the museum. Although initially perceived as marginal, these channels have gain centrality within the museum communication strategy and are activating a transformation in the way contents are conceptualized, planned and edited.

Current status of social media is changing the public's way of perceiving them from spaces of information to spaces of cultural broadcasting and social participation. As a consequence these tools are somehow affecting all areas of the museum not only the department of communication.

We want here to recount our experience managing these social media tools. Thus, while allowing us to broadcast who we are and what we do, these tools have also let us to listen to our audiences and reach to a wider public getting them increasingly involved in our activities. 
Finally, the text will briefly reviewed some aspects that are conditioning the management of these on-line channels. We believe, such aspects will be crucial to face the near future. Namely, these are the increase of actions to allow on line participation of the public, and the planning and production of digital contents by the scientific and educational teams of the museum. Both represent a challenge involving the museum adventuring itself in non-traditional territories for heritage communication.

Keywords: museum, popular culture, cultural broadcasting, digital contents, social media, cultural heritage 


\section{Introducción. L'ETNO. Museu Valencià d'Etnologia}

L'ETNO. Museu Valencià d'Etnología es una institución inaugurada en 1982 dirigida a la conservación, estudio y divulgación del patrimonio material e inmaterial vinculado a la cultura tradicional valenciana. Así mismo es una institución dedicada al estudio y la difusión de las dinámicas de la cultura y su diversidad, y como unidad integrante de la Diputación de Valencia, colabora con las iniciativas locales de patrimonio etnológico.

El objeto de estudio -la cultura popular y tradicional- se vertebra entorno diferentes ejes temáticos. Por un lado, trata lo que nos identifica como sociedad valenciana y aquello que se ha adoptado como propio procedente de otras culturas. Por otro, se refiere a la forma de vida de los valencianos y valencianas desde finales del mundo preindustrial hasta la actualidad: el final de las sociedades agrarias, los procesos de industrialización y urbanización y el impacto de modernización y la globalización...

Para desarrollar esta labor, el museo ha construido un mensaje que abandona la forma nostálgica de presentar su colección y sus exposiciones, y que apuesta por tratar el pasado desde el presente, una decisión no entendida muchas veces por un público interesado en ver un museo de costumbres y objetos de "ayer". Esta estrategia permite acercarnos a la sociedad tradicional desde formas y comportamientos actuales, partiendo de temas cercanos -significativos para la ciudadanía-, y generando interrogantes que permiten reflejar una sociedad -la pasada- con otra la actual-. Una forma de trabajar que no solo valora el papel del museo en tanto que institución que crea valor mas allá de su propia existencia, sino que genera discursos sobre temas cercanos y cotidianos incrementando su utilidad e interés para el público (Seguí, 2015:45).

Para entender mejor el trabajo realizado desde las redes sociales, entendidas como redes de comunicación que facilitan la expansión del museo y que llaman la atención de las personas de su entorno (Moreno, 2019: 100), conviene indicar de forma sucinta las áreas y los programas más destacados del museo, ya que ambos aspectos forman la "materia prima" del trabajo de las redes sociales del museo.

El museo dispone de casi todas las áreas de trabajo que componen este tipo de instituciones. Cuenta con departamentos de investigación sobre cultura tradicional, espacios de conservación y restauración de sus colecciones, una sección de didáctica y actividades educativas, una biblioteca y centro de documentación, una unidad de difusión y comunicación y un grupo de trabajo de producción de exposiciones. Asimismo, dispone de un área de trabajo dirigida al asesoramiento y cooperación con los museos y centros locales de patrimonio etnológico. Esta estructura se completa con el Museu de la Paraula, un museo de carácter virtual que muestra testimonios sobre la vida cotidiana de personas, en un recorrido temporal que comienza con entrevistas a personas nacidas antes de la Guerra Civil Española ${ }^{1}$.

\footnotetext{
${ }^{1}$ Ver http://www.letno.es/ y http://www.museudelaparaula.es/web/home/
} 
Presentar el pasado desde el presente es como hemos indicado la forma que el L'ETNO ha elegido para tratar la cultura tradicional. Esta forma de enfocar el discurso museológico se plasma en la mayoría de los programas que desarrolla el museo. Una muestra de ello son los ciclos culturales Espanta la Por o ETNOMUSIC, dos programas que nos acercan a la tradición desde lecturas actuales y contemporáneas ${ }^{2}$. El resto de la programación ofrece la habitual cartera de actividades de este tipo de instituciones (programas de conciertos, talleres, conferencias, proyecciones, presentaciones de libros, actividades formativas) y se organiza entorno a los siguientes contenidos: la colección, los temas tratados en las exposiciones temporales, el ciclo festivo anual, el contenido de la muestra permanente y la cultura tradicional.

El programa de exposiciones temporales combina diferentes líneas de actuación relacionadas con la misión principal del museo: exposiciones sobre fenómenos característicos de nuestra trama cultural y asociativa (la fiesta del Corpus, las bandas de música, muixerangues, pilota valenciana, Fallas), muestras que nos acercan a la sociedad valenciana tradicional a través de exhibiciones fotográficas o temas específicos (València en Blanc i Negre, indumentaria tradicional...), producciones de carácter antropológico y muestras de carácter histórico.

L'ETNO. Museu Valencià d'Etnologia ha inaugurado recientemente la exposición permanente No és fàcil ser valencià/na que trata la complejidad de la identidad valenciana desde una visión descriptiva y abierta «partiendo del debate existente entre un mundo culturalmente cada vez más homogéneo y el deseo de mantener las costumbres y prácticas que consideran propias y que les arraigan a un territorio y a una colectividad» ${ }^{3}$. La muestra aborda esta cuestión desde tres ámbitos geográficos: la ciudad y la relación entre lo global y lo local; las huertas y marjales como espacios de imaginarios y tópicos valencianos; y el secano y la montaña, ámbito de las invisibilidades, fenómenos sociales existentes pero invisibles para la población urbana. Desde el punto museográfico y discursivo, la exposición incorpora las nuevas formas de presentar las colecciones y sigue la línea marcada por los planteamientos instituciones europeas de carácter etnológico o antropológico que han cambiado la forma de exponer la cultura tradicional (Segui, 2015:40).

Hasta aquí hemos realizado un breve repaso a los elementos constitutivos del museo, es decir hemos analizado los qués (la función principal del museo, las áreas de trabajo y los programas) y el cómo, indicando de qué forma entendemos el estudio y divulgación de la sociedad valenciana tradicional. En este momento se hace necesario abordar el para quién: quiénes son los públicos del museo. La bibliografía sobre análisis de públicos es extensa y abundante para

\footnotetext{
${ }^{2}$ El programa Espanta la por es un ciclo de actividades realizado durante la Fiesta de Todos los Santos dirigido a divulgar el imaginario tradicional sobre el miedo y valorizarlo con respecto a fenómenos mas globales como la Fiesta de Hallowen. ETNOMUSIC es un programa de música folk y del mundo que muestra propuestas tradicionales y nuevas lecturas de la música tradicional. http://www.letno.es/

${ }^{3}$ http://letno.es/es/content/no-es-facil-ser-valencia
} 
los museos en general, sin embargo, no lo es tanto para los museos etnológicos o antropológicos, sorprendentemente los más numerosos de nuestro territorio ${ }^{4}$.

Los museos son instituciones multiservicio y por tanto tienen tipos de públicos muy diversos. En nuestro caso tenemos público familiar, adulto, experto (investigador) escolar, asociativo, multicultural, interesado por la cultura popular, por la diversidad... Un conjunto de personas que viene al museo en busca de formación/educación, ocio cultural o entretenimiento. Cada uno de ellos presenta una serie de características socioeconómicas diferentes. Con el objetivo de saber más sobre las personas que visitaban nuestras exposiciones, durante el año 2018, l'ETNO. Museu Valencià d'Etnologia realizó una encuesta para analizar la tipología y satisfacción de sus públicos (Cabañés, 2021: 74). Los datos obtenidos nos indican que el museo es parecido a otros museos antropológicos o etnológicos. Nuestros visitantes tienen alrededor de 50 años con la misma proporción entre hombres y mujeres y con una escasa presencia de visitantes jóvenes (18 a 24 años). En su mayoría están ocupados o jubilados, y tienen unos altos niveles de formación. El estudio nos indicaba que la mayoría de los visitantes asistían al museo por recomendación y que las características de la muestra/exposición visitada configuraban un tipo diferente de visitantes: las muestras de tipo costumbrista y nostálgico (fotográficas) presentaban una mayor afluencia de personas de mayor edad (mayores de 55 años) y de menor formación. Aspecto que contrastaba con el público de la muestra permanente que antes de su remodelación ya presenta una museografía moderna y contemporánea y que era visitada por grupos de públicos más jóvenes y con mayor nivel formativo ( 35 a 44 y 45 a 55 años).

\section{Trayectoria de las redes sociales de L'ETNO. Museu Valencià d'Etnologia}

L'ETNO puso en marcha sus redes sociales en el año 2012, momento en el que casi todos los museos y centros culturales de su entorno comenzaron a abrir este tipo de canales. Desde el principio hubo entidades como el MUVIM, la Rambleta o el IVAM que rápidamente consiguieron cifras de seguidores muy significativas. La causa de este crecimiento puede explicarse por el "tirón" de la marca de algunas de estas instituciones, y por la forma entender este fenómeno como un apartado básico de la comunicación de estas entidades, lo que les hizo apostar por la creación de equipos encargados de estas tareas.

Desde 2012, año en el que L'ETNO abrió sus perfiles de YouTube y Flickr, el museo ha entrado en Facebook, Twitter, Issuu (2013), Pinterest (2015) e Instagram (2017). De todos estos canales, cuatro mantienen una actividad periódica (Facebook, Instagram, Twitter, Youtube) y los otros tres permanecen abiertos, pero por motivos de carácter organizativo no están actualizados (Flickr, Pinterest, Issuu). Recientemente el museo ha revitalizado su canal de Spotify con la creación de listas musicales asociadas a sus actividades y publicaciones.

\footnotetext{
${ }^{4}$ Estadística de museos y colecciones museográficas 2018. Ministerio de Cultura y Deporte. 2020.
} 
El museo tiene un blog (http://etnobloc.es/), que depende la biblioteca, que se actualiza varias veces por semana con secciones fijas como las novedades bibliográficas, la presentación de un libro semanal, la información de las actividades de la biblioteca, etc.

Aunque el museo se desenvuelve en una temática menos propicia para las RRSS y no goza de un público objetivo tan aficionado a las redes sociales como otros centros culturales de la ciudad, la trayectoria en redes de l'ETNO presenta valores positivos en relación con otras instituciones de la misma temática y dimensión, por ello ha merecido el premio \#ConnectaMuseu a la institución que mejor trabajó las redes durante el $2018^{5}$.

\subsection{Objetivos y contenidos}

Las redes sociales del museo se valoraron, en principio, como unos canales a incorporar al trabajo del área de comunicación, por lo que se concibieron como espacios básicamente informativos. La puesta en marcha de estos nuevos canales respondio a los siguientes objetivos:

- Tener presencia en las redes sociales más populares emulando la tendencia de la mayoría de los centros culturales (Facebook, Twitter, Instagram)

- Infomar de nuestras actividades, de los trabajos y de las áreas del museo.

- Crear audiencia digital del museo. Generar una comunidad de seguidores en redes respecto de la actividad y función del museo.

Para conseguir estos objetivos se estableció una línea editorial que comprendía tres aspectos: los contenidos, el calendario de publicaciones y la estrategia principal de actuación. Resulta interesante observar cómo en estas primeras fases no era tan importante valorar la escucha y la participación de los seguidores, dos aspectos importantes que ofrecen las redes. Es importante resaltar que los destinatarios de estos canales tampoco interactuaban en aquellos años de la misma forma como lo hacen en la actualidad.

Desde el punto de vista de los contenidos, las redes sociales de l'ETNO abarcan 4 tipos de temas $^{6}$.

- La actividad del museo: información detallada de las actividades del museo para propiciar la asistencia del público a estas actividades.

- La función del museo. Qué es L’ETNO y para qué sirve. Una de las aportaciones más significativas de las redes sociales a la dinámica comunicativa del museo fue la posibilidad de abrir las áreas de trabajo al público. Estos canales nos permitían "convertir en noticia" las actividades de cada departamento del museo de forma

\footnotetext{
${ }^{5}$ Para conocer la evolución de las redes sociales de las instituciones culturales valencianas ver los informes anuales \#CONNECTAMUSEU realizado por La Visible. Cultura en movimiento. Esta empresa cada año otorga el premio \#ConnectaMuseu, reconocimiento a la institución con mejor trabajo en sus redes sociales. https://www.lavisible.org/.

${ }^{6}$ Todos estos temas tienen sus hashtags específicos y siempre se procura que tenga el acrónimo del museo: \#LETNOPropDeTu \#DescobreixLETNO \#LETNORestauració \#LETNOEnFamília \#EtnoXaxa \#EtnoObjecte \#Etnobiblio, etc.
} 
periódica, aspecto que nos ayudaba a valorizar el trabajo a veces poco conocido de los profesionales que trabajan en estos servicios (personal de restauración, educación, conservación o investigación, etc).

- Informar de aquello que hacen las instituciones que trabajan con nosotros. Aunque el museo ya contaba con una buena red de instituciones de carácter académico y cultural, así como una red consolidada de museos etnológicos locales, la puesta en marcha de las redes nos permitió incrementar las alianzas a un mayor número de instituciones y fortalecer las relaciones con el tejido cultural e institucional del museo.

- La cultura tradicional valenciana. Mas allá de sus actividades y programas, los museos son instituciones expertas que pueden y deben generar opinión e información sobre el tema que tratan. Sin embargo, esta faceta se realiza en muchas ocasiones a demanda de medios de comunicación, instituciones o particulares. Las redes nos brindaron la posibilidad de tratar la temática etnológica desde un punto de vista mas amplio y nos convirtieron en un espacio de información generalista (y experta) sobre temas relacionados con la cultura tradicional ${ }^{7}$.

Estos paquetes de contenidos se organizaron en un calendario semanal a modo de publicación periódica, en el que se concretaban cada una de las publicaciones, los recordatorios, la adjudicación de temas por días, el alcance y tratamiento de los eventos mas importantes (ciclos culturales, exposiciones, presentaciones, etc) y la forma de presentar estos eventos en cada una de las redes.

Para conseguir los objetivos propuestos se optó por una estrategia que podríamos definir como estrategia de saturación, consistente en publicar mucho y de forma muy periódica en cada perfil, repicando contenidos entre ellos y utilizando la web como espacio de apoyo de la información publicada. Esta forma de actuar nos permitía ganar visibilidad y crearnos espacio en un nuevo medio en el que nuestros visitantes comenzaban a transitar. Respecto a los públicos, aspecto relevante a la hora de trazar un plan de comunicación, el museo no consideró oportuna una segmentación explicita de sus publicaciones. Se prefiró que cada canal, asumiera estas publicaciones según sus perfiles principales de usuarios.

Los resultados después de ocho años de trabajo se pueden observar en el siguiente gráfico.

\footnotetext{
${ }^{7}$ Al respecto, es importante señalar que el canal de twitter del museo se creó con el nombre de @ valenciaetno, en un intento de hacer de este canal no solo un espacio de información del museo de etnología sino de la etnología y la cultura tradicional en el ámbito valenciano.
} 


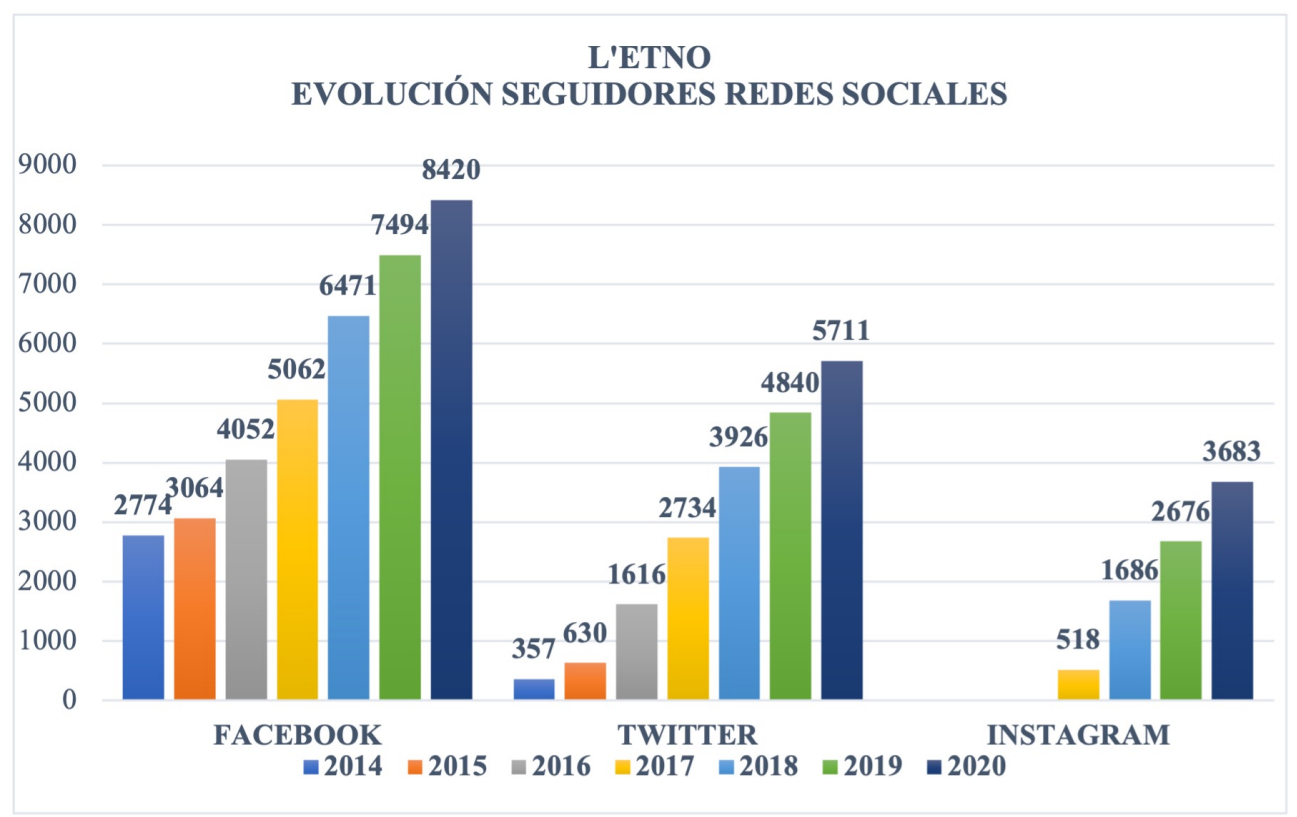

Fig. 1 Evolución de los seguidores en redes sociales

Los datos nos muestran un número significativo de seguidores. En Facebook, se ha producido un incremento del 300\% de seguidores en los últimos 7 años, con crecimientos absolutos de 100 seguidores mensuales cada año. Twitter presenta un porcentaje de incremento mayor (los seguidores se han multiplicado por 15) aunque partía de una situación casi residual. El incremento de Instagram es también muy interesante: se ha multiplicado por 6 en los últimos 4 años. Lo más destacable es el grado de seguimiento y participación de cada una de ellas -con niveles del $8 \%$ de engagement en el caso de Facebook ${ }^{8}$, y el ritmo de crecimiento de los seguidores, que se sigue manteniendo con el paso de tiempo de forma inalterable: 100 seguidores nuevos cada mes, en cada una de las tres redes.

A la vista de la trayectoria de estos últimos años podemos afirmar que los objetivos propuestos se han conseguido, ya que, salvando las distancias con otras entidades culturales de la ciudad (Informe \#ConnectaMuseu 2018), el museo goza de una presencia consolidada en redes sociales y dispone de una comunidad de seguidores estable y en crecimiento sostenido.

\subsection{Repercusiones organizativas tras 9 años de presencia en redes sociales}

El trabajo desarrollado durante estos años nos ha permitido comprobar que nuestra presencia en las redes es cada vez más necesaria, no solo por nuestro interés de informar, sino por la necesidad de trasladar parte de nuestro museo a un espacio en que el se ubica el consumidor de productos

${ }^{8}$ https://www.lavisible.org/wp-content/uploads/2019/12/Informe-Connecta-Museu-2018-lavisible.pdf. Pág 7. 
culturales (Celorrio, 2015:43). Esta apertura ha producido algunas modificaciones en el área de comunicación del museo. En primer lugar, se ha producido cierta mutación respecto del papel de los diferentes canales de información. Las redes han ampliado la comunicación con nuestros públicos y nos han permitido mantener de forma permanente la presencia social, desplazando en cierta forma a los medios tradicionales que cada vez ocupan un papel más institucional aunque no menos importante (notas de prensa, ruedas de prensa, newsletter).

Asimismo, dentro museo se ha entendido que las redes ya son elementos sustanciales de la comunicación y que es necesario dotarlas de personal especifico y de asignaciones presupuestarias estables para mantener los objetivos y los ritmos de publicación ${ }^{9}$. Por su parte los técnicos de las distintas áreas del museo han entendido que las redes sociales son muy eficaces para la imagen del museo y canales muy adecuados para mostrar su trabajo.

L'ETNO también ha propiciado la participación de los seguidores a través de campañas de dirigidas a generar fidelización de los públicos respecto de las propias redes, incentivar la visita a nuestras instalaciones, presentar las colecciones o tratar temas asociados con la exposición permanente $^{10}$.

Por último, el museo ha asumido que las redes también son instrumentos de escucha, en los que la información tiene un carácter bidireccional. Esta cuestión puede resultar obvia, pero en ciertas instituciones representa un cambio de funcionamiento a la hora de entender la relación con la ciudadanía. Las instituciones públicas están acostumbradas a trabajar desde el rol de "emisor" en la relación comunicativa y los museos en muchas ocasiones participan de esta forma de actuar. Son espacios de estudio y divulgación, servicios desde los que emana el saber hacia la sociedad, con pocos cauces para recoger iniciativas y opiniones de los usuarios. La elevada frecuencia de publicaciones adoptada por l'ETNO ha aumentado la frecuencia de sugerencias, opiniones y preguntas, que ha hecho necesaria la elaboración de cierto protocolo para atenderlas con prontitud y franqueza.

\section{Nuevas propuestas. Las redes sociales de l'ETNO como divulgadoras de contenidos}

La actividad generada en redes sociales ha expandido el museo hacia un territorio en el que habita una comunidad online compuesta por públicos que nos visitan y públicos que sólo nos siguen en el entorno digital. Es necesario llenar de contenidos ese espacio para propiciar el interés de unos y otros, retroalimentar ambas experiencias, y enriquecer las actividades presenciales gracias a los contenidos digitales (Celaya y Saldaña, 2013: 24).

\footnotetext{
${ }^{9}$ L'ETNO destina a las redes sociales y digitalización un porcentaje presupuestario semejante a la mayoría de museos, que se sitúa entre el 1\% y 5\%. Ver Museos, profesionales de los museos y COVID-19. ICOM. Pág 10 .

${ }^{10}$ ETNOBJECTE-, \#CapAUnMuseuSosteniblE; \#WikiLovesFolkFalles \#LaCiutatEmmascarada
} 
Desde l'ETNO, somos conscientes que la estrategia digital debe impregnar todas las áreas del museo y que es necesario dotarse de tecnologías y aplicaciones que permitan la digitalización de contenidos y su acceso virtual. Este esfuerzo requiere la adscripción de unos recursos que en muchas ocasiones no están disponibles. Por ello, las iniciativas que aquí detallamos afectan solo al espacio de las redes sociales y a sus posibilidades para divulgar los contenidos del museo.

El primer gran objetivo de esta nueva etapa es aprovechar el potencial de las redes para generar participación e interacción de los usuarios. Entendemos que el museo tiene suficiente comunidad y trayectoria para dirigir esta relación hacia propuestas en las que los seguidores usen las redes para "saber más" sobre nuestra cultura tradicional, nuestra exposición y nuestra colección. Este objetivo se desarrollará por "capas". Como se ha comentado al inicio de este texto los públicos del museo son diversos y la aportación de contenidos debe atender a sus diferentes expectativas. Por ello los contenidos trabajarán diferentes niveles/capas de complejidad: desde propuestas de corta duración de carácter desenfadado dirigidas presentar el museo como una institución menos seria y próxima; la elaboración de videos de carácter divulgativo para presentar muestras o colecciones; y la muestra del trabajo de expertos o procesos y técnicas de producción agrícola o industrial.

El otro gran objetivo de esta etapa es convertir las redes sociales en espacios más centrados en los contenidos. Esta labor se desarrollará a través de los siguientes apartados:

- Dar voz a las colecciones. Desarrollar videos de pequeño y gran formato que muestren las piezas de la colección, su procedencia, uso, y características. No se trata tanto de invertir en la digitalización y muestra en tres dimensiones de los objetos de nuestra colección como que un conservador nos explique su uso y origen. El museo, como se ha indicado no dispone de objetos "singulares" a diferencia de lo museos de arte. L'ETNO atesora objetos cotidianos que generan valor por su componente social, el desarrollo de una técnica o proceso concreto, etc. (\#SabeuQue, \#RebostDLetno, \#QuèÉsEtnologia). En este terreno también se pondrán en marcha juegos que provoquen la participación de los seguidores (\#LETNOTrivial).

- Dar voz a las áreas de trabajo del museo. Hasta ahora las redes de l'ETNO han presentado de forma periódica las actividades que realiza cada área del museo. El objetivo de esta nueva fase es presentar los procesos y procedimientos que se desarrollan en cada una de ellas y los profesionales que las llevan a cabo. El abanico de temas es muy amplio: el trabajo con las colecciones, la preparación y elaboración de trabajos de restauración, el trabajo de campo o la preparación de programas de carácter educativo y su puesta en macha.

- Incrementar la producción de videos sobre procesos agrícolas e industriales de la sociedad valenciana durante el siglo XX. Muestras que nos permitan divulgar las formas y los usos de la sociedad tradicional: procesos artesanos, rituales populares, manifestaciones festivas, etc.

- Continuar el trabajo de divulgación del contenido de las exposiciones temporales, a través de visitas guiadas y aportaciones de los comisarios e investigadores, en las que plasme además una visita a la muestra, el proceso de su producción. 
- Convertir las redes en canales de comunicación entre el visitante y el experto. Algunos museos están usando aplicaciones para generar una comunicación directa entre el equipo del museo y los visitantes a las salas, que pueden preguntar cuestiones suscitadas en la visita ${ }^{11}$. Este trabajo se podrá realizar desde las redes sociales o desde el canal de WhatsApp que el museo ha puesto en marcha en febrero 2021. (\#PreguntaALETNO).

- Valoración de la visita por parte del visitante. Pondremos en marcha campañas en redes que el las que el visitante pueda valorar la experiencia de la visita al salir de las salas de exposición. El objetivo es provocar esta participación a través de cauces que permitan cierta monitorización en el que los usuarios se vean recompensados (Celaya y Saldaña, 2013: 18).

Todas estas iniciativas superan las atribuciones del área de comunicación del museo y ponen el acento en la necesidad de que todas las áreas de museo se impliquen en la labor digital de la institución, ya que para ofrecer productos y relaciones de calidad con la comunidad online no son suficientes las maneras y las dinámicas estrictamente comunicativas y es necesario que las áreas de conservación, protagonistas de los contenidos de los museos, comprendan su importante labor.

\section{Conclusiones}

La trayectoria de las redes sociales de l'ETNO puede resultar de ejemplo (modesto) para analizar el peso y la trascendencia que las redes sociales han tenido en las organizaciones culturales y más concretamente en los museos. Un recorrido que empezó entendiento las redes como espacios de meramente informativos y que nos ha abierto las puertas a un entorno propio en el que hay que mantener una estrecha relacion con una audiencia digital que debe ser informada, atendida, escuchada y que está acostumbrada a la partipación, a la valoración y a la bidireccionalidad. Este escenario nos ha hecho cambiar la manera de entender nuestra forma de comunicar y nos ha obligado a crear estructuras estables que atiendan lo que ocurre en su interior.

En la actualidad, l'ETNO debe abrir una nueva etapa dirigida a proporcionar más valor a lo que ponemos en el ámbito digital y asi ser mas competitivos respecto de otras instituciones. Esta etapa transcurre necesariamente por la creación de contenidos para el entorno digital que puedan ofrecerse a través de las redes sociales. En este nuevo rol se hara necesario que todo el museo, y en especial las áreas de conservación y producción, asuma la necesidad de atender a los públicos digitales.

\footnotetext{
${ }^{11}$ El Brooklyn Museum, Brooklyn, E.U, ha generado una apliación (“Ask”) dirigida a propiciar la participación del visitantes a través de preguntas al equipo de expertos del museo. (Encuentro Museo digital. Futuros y posibilidades, organizado por Fundación Telefónica. México A.C. y MUAC. Museo Universitario de Arte Conteporáneo 2017. Pág 183.)
} 


\section{Referencias}

Arguello, G. (2017): "Estudios de caso". En Encuentro Museo digital. Futuros y posibilidades. Fundación Telefónica. México A.C. y MUAC. Museo Universitario de Arte Conteporáneo 2017. Museo Universitario Arte Contemporáneo, UNAM. 149-195. $<$ https://www.fundaciontelefonica.com/cultura-digital/publicaciones/museo-digital/684/> [Consulta: diciembre de 2021]

CABAÑÉS MARTINEZ. F. (2021): “Estudio de públicos de L'ETNO. Museu Valencià d'Etnologia. Informe preliminar”. ICOM España. En Los habitantes del museo. Actas del II Encuentro de Museologia - Valencia 2018. ICOM España (Comité Español del Consejo Internacional de Museos). 74-99.

Celaya, J. y Saldaña, I. (2013): Los museos en la era digital. Uso de nuevas tecnologías antes, durante y después de visitar un museo, centro cultural o galería de arte.DOSDOCE. $<$ https://www.igartubeitibaserria.eus/es/files/los-museos-en-la-era-digital $>$ [Consulta: diciembre de 2020]

Celorrio Moreno, I. (2015): La difusión del museo en el entorno digital: nuevos recursos tecnológicos y online. Tesis doctoral. Valladolid: Universidad de Valladolid. Facultad de Ciencias Sociales, Jurídicas y de la Comunicación. $<$ http://uvadoc.uva.es/bitstream/handle/10324/14103/TFG-

N.331.pdf? sequence $=1$ \&isAllowed $=\mathrm{y}>$ [Consulta: enero 2021]

ETNO. Museu Valencià d'Etnologia (2021): Memòria d'activitats 2020.

Estadística de museos y colecciones museográficas 2018. Ministerio de Cultura y Deporte. 2020. $<$ http://www.culturaydeporte.gob.es/dam/jcr:86600fdd-26b3-430b-95a5-

aba45b4fb3c2/estadistica-de-museos-y-colecciones-museograficas-2018.pdf> [Consulta: febrero 2021]

Gómez VILChEZ, S. (2012): Museos españoles y redes sociales, Evaluación de preferencia y participación en Revista Telos núm 90. Fundacion Telefonica. $<$ https://telos.fundaciontelefonica.com/archivo/numero090/museos-espanoles-y-redessociales/> [Consulta: en enero 2021]

$\operatorname{ICOM}$ (2020): Museos, profesionales de los museos y COVID-19. ICOM. Consejo Internacional de Museos $<$ https://icom.museum/wp-content/uploads/2020/05/Informe-museos-y-COVID19.pdf $>$ Consulado en diciembre 2020

Informe Connecta Museu tendencias de comunicación en las redes sociales de museos y centros culturales valencianos 2018. La Visible. Cultura en movimiento $<$ https://www.lavisible.org/wp-content/uploads/2019/12/Informe-ConnectaMuseu-2018-lavisible.pdf> [Consulta: febrero de 2021] 
MAteos Rusillo, S. (2012): Manual de comunicación para museos y atractivos patrimoniales. Gijón. Editorial Trea.

Moreno SÁncheZ, I. (2019): "Museística y nuevas redes digitales: en busca de la interafectividad con todas las personas" en Informe sobre el Estado de la Cultura en España. Cultura local, democracia, desarrollo. 2019. Fundación Alternativas. Observartorio de Cultura y Comunicación. Madrid. <https://www.fundacionalternativas.org/laspublicaciones/informes/el-estado-de-la-cultura-en-espana-2019-cultura-local-democraciadesarrollo $>$ [Consulta: enero de 2021]

SEguí SEguí. J. (2015): “Esta exposición no es para este museo. Las salas permaentes del Museu Valencià d'Etnologia" en El desafio de exponer, procesos y retos museológicos. Bilbao. Servicio Editorial de la Universidad del Pais Vasco. 